\title{
REDUCIBILITY OF ISOMETRIC IMMERSIONS
}

\author{
JOHN DOUGLAS MOORE
}

\begin{abstract}
For $i=1,2$, suppose that the connected riemannian manifold $M_{i}$ possesses a codimension $p_{i}$ euclidean isometric immersion whose first normal space has dimension $p_{i}$ and whose type number is at least two at each point, and let $N=\operatorname{dim}\left(M_{1} \times M_{2}\right)+$ $p_{1}+p_{2}$. In this note it is proven that if $f$ is any isometric immersion from the riemannian product $M_{1} \times M_{2}$ into euclidean $N$-space $E^{N}$, then there exists an orthogonal decomposition $E^{N}=E^{N_{1}} \times E^{N_{2}}$, together with isometric immersions $f_{i}: M_{i} \rightarrow E^{N_{i}}$ such that $f=f_{1} \times f_{2}$.
\end{abstract}

An isometric immersion $f$ from a riemannian product $M_{1} \times M_{2}$ into $N$-dimensional euclidean space $E^{N}$ is said to be reducible if there is an orthogonal product decomposition $E^{N}=E^{N_{1}} \times E^{N_{2}}$ together with isometric immersions $f_{1}: M_{1} \rightarrow E^{N_{1}}$ and $f_{2}: M_{2} \rightarrow E^{N_{2}}$ such that $f=f_{1} \times f_{2}$. It is known that if $M_{1}$ and $M_{2}$ are connected and their Riemann-Christoffel curvature tensors are nonzero almost everywhere, then every codimension two euclidean isometric immersion of $M_{1} \times M_{2}$ is reducible [1], [5]. This note is devoted to a more general reducibility theorem.

To formulate the hypothesis for our theorem, we let $V$ denote the tangent space to a riemannian manifold $M$ at a point $m$. The RiemannChristoffel curvature tensor $R$ at $m$ can be regarded as an endomorphism of $V \wedge V$ which is symmetric with respect to the inner product defined by the riemannian metric. We will say that $M$ satisfies condition $A(p)$ at $m$ if there exist vectors $u, v \in V$ such that $R(u \wedge v)$ has rank at least $2 p$. (Recall that $R(u \wedge v)$ has rank $2 p$ iff $p$ is the largest integer such that $R(u \wedge v) \wedge \cdots \wedge R(u \wedge v)(p$ times $) \neq 0[3$, p. 55].)

REDUCIBILITY THEOREM. For $i=1,2$, let $M_{i}$ be a connected riemannian manifold which satisfies condition $A\left(p_{i}\right)$ almost everywhere, and let $N=$ $\operatorname{dim}\left(M_{1} \times M_{2}\right)+p_{1}+p_{2}$. Then any isometric immersion of the riemannian product $M_{1} \times M_{2}$ into $E^{N}$ is reducible.

Notice that if $M_{i}$ possesses a codimension $p_{i}$ euclidean isometric immersion $f_{i}$ whose first normal space has dimension $p_{i}$ and whose type number is at least two at each point (in the sense of Allendoerfer [2], [4, pp. 349-354]), then $M_{i}$ satisfies condition $A\left(p_{i}\right)$ everywhere. In this

Presented to the Society, August 10, 1971; received by the editors October 5, 1971. AMS 1970 subject classifications. Primary 53B25; Secondary 53C40.

Key words and phrases. Isometric immersion, riemannian product, type number.

(c) American Mathematical Society 1972 
case the dimension $N$ in the Reducibility Theorem is clearly optimal, because if $g: E^{N} \rightarrow E^{N+1}$ is a cylindrical isometric immersion, $g \circ\left(f_{1} \times f_{2}\right)$ : $M_{1} \times M_{2} \rightarrow E^{\mathrm{V}+1}$ will seldom be reducible. Reducibility is an uncommon phenomenon except in the lowest possible codimension.

$M$ satisfies condition $A(1)$ wherever $R$ is nonzero, i.e. at the nonflat points of $M$. Thus an induction based on the Reducibility Theorem and the following lemma will yield a generalization of Theorem 1 in [5].

LEMMA 1. If the riemannian manifold $M_{i}$ satisfies condition $A\left(p_{i}\right)$ at $m_{i}$ for $i=1,2$, then $M_{1} \times M_{2}$ satisfies condition $A\left(p_{1}+p_{2}\right)$ at $\left(m_{1}, m_{2}\right)$.

To prove the lemma we note that the tangent space $V$ to $M_{1} \times M_{2}$ at $\left(m_{1}, m_{2}\right)$ possesses an orthogonal direct sum decomposition $V=V_{1} \oplus V_{2}$, where $V_{i}$ consists of the vectors tangent to $M_{i}$. We regard $V_{i} \wedge V_{i}$ as a subspace of $V \wedge V$. If $R_{i}: V_{i} \wedge V_{i} \rightarrow V_{i} \wedge V_{i}$ is the curvature tensor of $M_{i}$ and $\pi_{i}: V \wedge V \rightarrow V_{i} \wedge V_{i}$ is the orthogonal projection, then

$$
R=R_{1} \circ \pi_{1}+R_{2} \circ \pi_{2}
$$

is the curvature tensor of $M_{1} \times M_{2}$. If condition $A\left(p_{i}\right)$ holds at $m_{i}$, then there exist vectors $u_{i}, v_{i} \in V_{i}$ such that $R_{i}\left(u_{i} \wedge v_{i}\right)$ has rank at least $2 p_{i}$. By (1),

$$
R\left(\left(u_{1}+u_{2}\right) \wedge\left(v_{1}+v_{2}\right)\right)=R_{1}\left(u_{1} \wedge v_{1}\right)+R_{2}\left(u_{2} \wedge v_{2}\right) .
$$

Since $R_{i}\left(u_{i} \wedge v_{i}\right) \in V_{i} \wedge V_{i}$, the sum on the right has rank at least $2\left(p_{1}+p_{2}\right)$, which proves that condition $A\left(p_{1}+p_{2}\right)$ is satisfied at $\left(m_{1}, m_{2}\right)$.

Let $n=\operatorname{dim}\left(M_{1} \times M_{2}\right)$ and $p=p_{1}+p_{2}$ so that $N=n+p$. An isometric immersion $f: M_{1} \times M_{2} \rightarrow E^{N}$ and a choice of orthonormal basis for the normal space to $M_{1} \times M_{2}$ at $\left(m_{1}, m_{2}\right)$ determine $p$ second fundamental forms $\Phi^{\lambda}, n+1 \leqq \lambda \leqq N$, at $\left(m_{1}, m_{2}\right)$. The $\Phi^{\lambda}$ 's are symmetric bilinear forms on the tangent space $V$ at $\left(m_{1}, m_{2}\right)$, and they determine symmetric endomorphisms $A^{\lambda}$ of $V$ by

$$
\left\langle A^{\lambda}(u), v\right\rangle=\Phi^{\lambda}(u, v) \text { for } u, v \in V,
$$

where $\langle$,$\rangle denotes the riemannian metric. The A^{\lambda}$,s in turn determine symmetric endomorphisms $A^{\lambda} \wedge A^{\lambda}$ of $V \wedge V$ which satisfy the Gauss equation

$$
R=\sum_{\lambda=n+1}^{N} A^{\lambda} \wedge A^{\lambda} .
$$

LEMMA 2. Suppose that the second fundamental forms $\Phi^{\lambda}$ of an isometric immersion f from a connected riemannian product $M_{1} \times M_{2}$ into $E^{N}$ have the following property at every point $\left(m_{1}, m_{2}\right)$ in $M_{1} \times M_{2}$ :

$$
\Phi^{\lambda}\left(w_{1}, w_{2}\right)=0 \text { for all } w_{1} \in V_{1}, w_{2} \in V_{2}, n+1 \leqq \lambda \leqq N .
$$

Then $f$ is reducible. 
This lemma is proven in $\S 2$ of [5]. In order to use Lemma 2 to prove the Reducibility Theorem it suffices by continuity to show that hypothesis (3) holds at almost all points in $M_{1} \times M_{2}$. By the hypothesis of the Reducibility Theorem and Lemma 1 it suffices to show that (3) holds at those points of $M_{1} \times M_{2}$ at which condition $A(p)$ is satisfied.

Assume now that $\left(m_{1}, m_{2}\right)$ is a point in $M_{1} \times M_{2}$ at which condition $A(p)$ holds. Then we can choose vectors $u, v$ in the tangent space $V$ at $\left(m_{1}, m_{2}\right)$ so that $R(u \wedge v)$ has rank at least $2 p$. By equation (2),

$$
R(u \wedge v)=\sum_{\lambda=n+1}^{N} A^{\lambda}(u) \wedge A^{\lambda}(v) .
$$

It follows that $R(u \wedge v)$ has rank exactly $2 p$ and that the $2 p$ vectors $A^{\lambda}(u)$, $A^{\lambda}(v), n+1 \leqq \lambda \leqq N$, are linearly independent. Now let $u=u_{1}+u_{2}$ and $v=v_{1}+v_{2}$, where $u_{i}, v_{i} \in V_{i}$. Using (1) and (2) we see that

$$
\sum_{\lambda=n+1}^{N} A^{\lambda}\left(u_{1}\right) \wedge A^{\lambda}(u)=\sum_{\lambda=n+1}^{N} A^{\lambda}\left(u_{1}\right) \wedge A^{\lambda}\left(u_{2}\right)=R\left(u_{1} \wedge u_{2}\right)=0
$$

and we conclude that the $A^{\lambda}\left(u_{1}\right)$ 's (and hence the $A^{\lambda}\left(u_{2}\right)$ 's) lie in the subspace of $V$ generated by the $A^{\lambda}(u)$ 's; in fact, by Cartan's lemma,

$$
A^{\lambda}\left(u_{1}\right)=\sum_{\mu=n+1}^{N} c_{\mu}^{\lambda} A^{\mu}(u), \quad c_{\mu}^{\lambda}=c_{\lambda}^{\mu}
$$

where the $c_{\mu}^{\lambda}$ 's are real numbers. Similarly we can show that the $A^{\lambda}\left(v_{1}\right)$ 's and the $A^{\lambda}\left(v_{2}\right)$ 's lie in the span of the $A^{\lambda}(v)$ 's. After a possible change of orthonormal basis for the normal space we can arrange that

$$
A^{\lambda}\left(u_{1}\right)=c_{\lambda} A^{\lambda}(u), \quad A^{\lambda}\left(u_{2}\right)=\left(1-c_{\lambda}\right) A^{\lambda}(u),
$$

where $c_{\lambda}=1$ for $n+1 \leqq \lambda \leqq q, c_{\lambda} \neq 0,1$ for $q+1 \leqq \lambda \leqq r$, and $c_{\lambda}=0$ for $r+1 \leqq \lambda \leqq N$. Equations (1), (2), and (4) now imply that

$$
\begin{gathered}
\sum_{\lambda=n+1}^{r} c_{\lambda} A^{\lambda}(u) \wedge A^{\lambda}\left(v_{2}\right)=\sum_{\lambda=n+1}^{N} A^{\lambda}\left(u_{1}\right) \wedge A^{\lambda}\left(v_{2}\right)=R\left(u_{1} \wedge v_{2}\right)=0, \\
\sum_{\lambda=a+1}^{N} A^{\lambda}\left(v_{1}\right) \wedge\left(1-c_{\lambda}\right) A^{\lambda}(u)=0 .
\end{gathered}
$$

Therefore $A^{n+1}\left(v_{2}\right), \cdots, A^{r}\left(v_{2}\right)$ and $A^{q+1}\left(v_{1}\right), \cdots, A^{N}\left(v_{1}\right)$ are in the span of the $A^{\lambda}(u)$ 's. But they are also in the span of the $A^{\lambda}(v)$ 's, and since the $A^{\lambda}(u)$ 's and the $A^{\lambda}(v)$ 's are linearly independent we must have

$$
A^{\lambda}\left(v_{2}\right)=0 \text { for } n+1 \leqq \lambda \leqq r, \quad A^{\lambda}\left(v_{1}\right)=0 \text { for } q+1 \leqq \lambda \leqq N .
$$


In particular the vectors $A^{\lambda}(v), q+1 \leqq \lambda \leqq r$, must vanish, and since these vectors are linearly independent, $q=r$.

We adopt the following index conventions: $n+1 \leqq \alpha \leqq q, q+1 \leqq \rho \leqq N$. Then $c_{\alpha}=1, c_{\rho}=0$, and it follows from (4) and (5) that

$$
A^{\alpha}\left(u_{1}\right)=A^{\alpha}(u), \quad A^{\alpha}\left(v_{1}\right)=A^{\alpha}(v), \quad A^{\rho}\left(u_{1}\right)=A^{\rho}\left(v_{1}\right)=0 .
$$

Hence if $w_{2} \in V_{2}$,

$$
\begin{gathered}
\sum_{\alpha} A^{\alpha}(u) \wedge A^{\alpha}\left(w_{2}\right)=\sum_{\lambda=n+1}^{N} A^{\lambda}\left(u_{1}\right) \wedge A^{\lambda}\left(w_{2}\right)=R\left(u_{1} \wedge w_{2}\right)=0, \\
\sum_{\alpha} A^{\alpha}(v) \wedge A^{\alpha}\left(w_{2}\right)=0 .
\end{gathered}
$$

Since the $2(q-n)$ vectors $A^{\alpha}(u), A^{\alpha}(v)$ are linearly independent, we can conclude that $A^{\alpha}\left(w_{2}\right)=0$. Similarly we can show that $A^{\rho}\left(w_{1}\right)=0$ when $w_{1} \in V_{1}$. Therefore

$$
\Phi^{\alpha}\left(w_{1}, w_{2}\right)=\left\langle w_{1}, A^{\alpha}\left(w_{2}\right)\right\rangle=0, \quad \Phi^{\rho}\left(w_{1}, w_{2}\right)=\left\langle A^{\rho}\left(w_{1}\right), w_{2}\right\rangle=0,
$$

for $w_{1} \in V_{1}, w_{2} \in V_{2}$. This establishes (3) at $\left(m_{1}, m_{2}\right)$ and finishes the proof of the Reducibility Theorem.

\section{REFERENCES}

1. S. Alexander, Reducibility of Euclidean immersions of low codimension, J. Differential Geometry 3 (1969), 69-82. MR 40 \#3467.

2. C. B. Allendoerfer, Rigidity for spaces of class greater than one, Amer. J. Math. 61 (1939), 633-644. MR 1, 28.

3. R. L. Bishop and R. J. Crittenden, Geometry of manifolds, Pure and Appl. Math., vol. 15, Academic Press, New York, 1964. MR 29 \#6401.

4. S. Kobayashi and K. Nomizu, Foundations of differential geometry. Vol. II, Interscience Tracts in Pure and Appl. Math., no. 15, vol. II, Interscience, New York, 1969. MR 38 \#6501.

5. J. D. Moore, Isometric immersions of riemannian products, J. Differential Geometry 5 (1971), 159-168.

Department of Mathematics, University of California, Santa Barbara, CALIFORNIA 93106 